\title{
'Doubling down' on the autophagy pathway to suppress tumor growth
}

\author{
Andrew M. Leidal and Jayanta Debnath ${ }^{1}$ \\ Department of Pathology, Helen Diller Family Comprehensive Cancer Center, University of California at San Francisco, \\ San Francisco, California 94143, USA
}

In this issue of Genes \& Development, Wei and colleagues (pp. 1204-1216) use elegant genetic approaches to simultaneously delete the essential autophagy gene FIP200 (FAK family-interacting protein of $200 \mathrm{kDa}$ ) and the signaling adaptor p62/SQSTM1 within established murine tumors, which reveals an unexpected synergism between the autophagy pathway and p62 in driving tumor growth. Intriguingly, these observations suggest that the combined targeting of autophagy and p62 may serve as an effective approach to treat specific cancers.

Macroautophagy (hereafter called autophagy) is a catabolic process in which cells sequester cytoplasmic proteins and organelles into double-membrane vesicles, called autophagosomes, and deliver them to the lysosome, where the contents are subsequently digested and recycled. The bulk degradation of cellular material through autophagy serves as an important quality control mechanism and survival pathway during starvation and stress (Kimmelman 2011). Given the prominent role of stress resistance and survival in the development and progression of cancer, it is not surprising that the autophagy pathway is emerging as an important determinant of tumor cell fate. However, current evidence indicates multifaceted roles for autophagy during tumorigenesis, either suppressing or promoting tumor growth, depending on the cancer subtype and the stage of tumor development (Kimmelman 2011; White 2012). To characterize the distinct functions of autophagy during tumor progression, separate from its role in tumor initiation, Wei et al. (2014) developed an inducible mouse model to ablate autophagy within established tumors and uncovered that the autophagy pathway cooperates with the signaling adaptor p62/SQSTM1 to drive tumor growth.

The core components of the autophagy machinery, called ATGs (for autophagy-related), are conserved from yeast to humans. One notable exception is FIP200 (FAK familyinteracting protein of $200 \mathrm{kDa}$ ), which lacks an obvious sequence ortholog within yeast but is critical for autophagy in higher eukaryotes. In mammals, FIP200 forms a complex

[Keywords: tumor growth; p62; autophagy; mouse models]

${ }^{1}$ Corresponding author

E-mail jayanta.debnath@ucsf.edu

Article is online at http://www.genesdev.org/cgi/doi/10.1101/gad.244681.114. with the serine-threonine kinase ULK1 and two additional proteins, ATG13 and ATG101, which together serve to regulate the early steps of autophagosome formation. Subsequently, additional ATGs mediate elongation of the autophagosome membrane and, ultimately, its closure to form the double-membrane vesicle. Selective autophagy receptors that bind LC3, of which the archetypal family member is p62/SQSTM1, are recruited with their cargo to the maturing autophagosome and sequestered from the cytosol upon closure of the double-membrane vesicle (Kimmelman 2011; Boya et al. 2013). Finally, the autophagosome fuses with the lysosome, and its contents are degraded. Importantly, disruption of essential genes within the autophagy pathway, including FIP200, impairs autophagosome biogenesis at its earliest stages and leads to the accumulation of substrates such as p62 (Fig. 1).

The first links between autophagy and cancer came with the discovery that beclin1 haploinsufficiency predisposes both humans and mice to tumor development (Kimmelman 2011). Subsequent studies in genetically engineered murine models (GEMMs) of cancer have revealed that defects in the autophagy pathway promote genome damage, inflammation, and cancer cell growth (White 2012). The molecular basis for these pro-oncogenic changes in part appears to be linked to the aberrant accumulation of the selective autophagy receptor p62, a multifunctional adaptor protein at the nexus of the NF-кB, NRF2-KEAP1, and mTOR signaling pathways (Fig. 1; White 2012). However, autophagy is not purely tumor-suppressive because numerous studies indicate that pathway inhibition can impede tumor growth and sensitize cancer cells to therapy. For example, tumors with activating mutations in RAS family proteins, such as lung and pancreatic cancer, rely on the autophagy pathway for growth and proliferation (Guo et al. 2013). Similarly, in GEMMs for hereditary breast cancer that harbor deletions in $P A L B 2$, the partial allelic loss of beclin1 increases apoptosis and delays tumor development in a p53-dependent manner (Guo et al. 2013). Previous studies by Guan and colleagues (Wei et al. 2011)

(C) 2014 Leidal and Debnath This article is distributed exclusively by Cold Spring Harbor Laboratory Press for the first six months after the full-issue publication date (see http://genesdev.cshlp.org/site/misc/terms.xhtml). After six months, it is available under a Creative Commons License (AttributionNonCommercial 4.0 International), as described at http://creativecommons. org/licenses/by-nc/4.0/. 


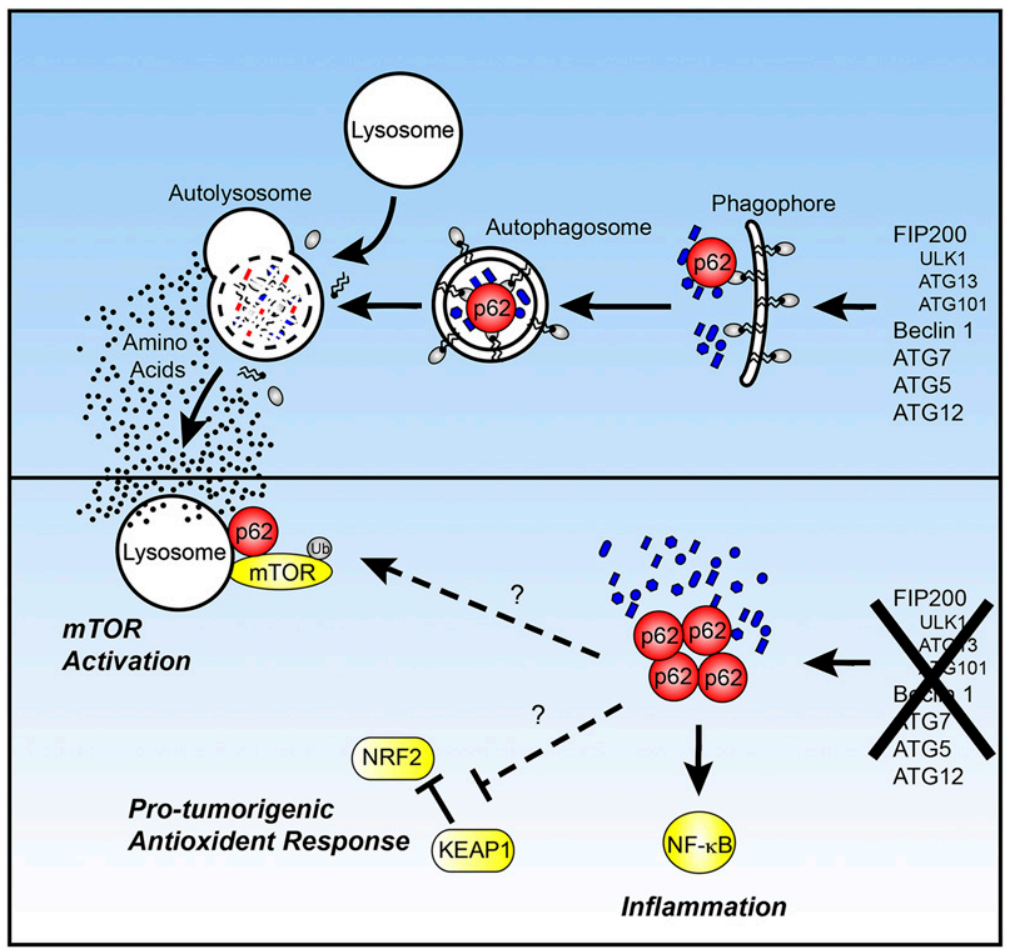

Figure 1. Autophagy regulates p62/SQSTM1 levels to control multiple pro-oncogenic signaling pathways. (Top panel) The formation of autophagosomes is controlled at the initiation stage by the coordinated action of the ULK1-FIP200-ATG13-ATG101 and Beclin1-Vps34 signaling complexes and enzymes, including ATG7 and ATG5-ATG12, that function to lipidate LC3. Selective autophagy receptors, including p62, interact with the autophagosome membrane and are sequestered from the cytosol along with their cargos upon membrane closure. Fusion of the autophagosome with lysosomes triggers the degradation of sequestered cargo, enabling the basic constituents of digested material to be recycled by the cells. (Bottom panel) Loss of essential autophagy genes, such as FIP200 or ATG7, attenuates the autophagy pathway and results in the accumulation of both selective autophagy receptors and cargo. Increased p62 potentiates NF-кB signaling and the elaboration of protumorigenic inflammatory cytokines. Defects in p62 clearance by the autophagy pathway may also reinforce pro-oncogenic signaling through the activation of mTOR and NRF2, which respectively control cellular growth and antioxidant response pathways. have also demonstrated that genetic ablation of FIP200 in a polyoma middle $\mathrm{T}$ antigen (PyMT)-driven breast cancer model profoundly inhibits the frequency and progression of murine mammary tumors . Finally, therapeutic inhibition of autophagy in diverse tumor types has shown promise as an effective anti-cancer treatment (Yang et al. 2011).

Overall, it remains unclear how autophagy inhibition leads to such seemingly disparate outcomes in different cancers. How do we determine which tumors will respond to autophagy inhibition? Fundamentally, the answers to these questions require a better understanding of how the autophagy pathway functions in specific contexts, including distinct tumor types, oncogenic backgrounds, or tumor stages. Perhaps one of the most significant challenges in assessing the function of autophagy in cancer has been an inability to discern between the contributions of autophagy to tumor initiation versus its role in tumor progression. To tackle this important issue, Wei et al. (2014) developed a clever genetic approach that allowed them to specifically ablate autophagy in advanced tumors. Mouse embryo fibroblasts (MEFs) derived from FIP200 floxed mice were transformed with $\mathrm{E} 1 \mathrm{~A} / \mathrm{H}-\mathrm{Ras}^{\mathrm{V} 12}$ and subsequently transduced with a retroviral vector encoding a tamoxifeninducible form of the Cre recombinase $\left(\mathrm{Cre}^{\mathrm{ERT}}\right)$; as a result, FIP200 could be conditionally deleted in established tumors in vivo upon administration of tamoxifen. Intriguingly, upon FIP200 deletion and impairment of the autophagy pathway in established tumors, Wei et al. (2014) observed a profound reduction in tumor growth. These observations were not exclusive to transformed MEFs, since deletion of FIP200 within tumors derived from PyMT transformed mouse mammary tumor cells also resulted in impaired growth. An assessment of the proportion of replicating and apoptotic cells within the two treatment groups revealed that the defect in tumor growth was a manifestation of both attenuated tumor cell proliferation and increased cell death. Although the investigators did not examine the status of metabolic pathways in the FIP200-null tumors, it is tempting to speculate that the impaired proliferation and increased apoptosis are at least partly associated with a failure to sustain energy and biosynthetic pathways (Guo et al. 2013).

Similar to previous studies examining the function of autophagy during tumorigenesis, Wei et al. (2014) observed a marked accumulation of p62 upon deletion of FIP200 (Mathew et al. 2009; White 2012). However, in stark contrast with those reports, p62 accretion in FIP200deficient tumor cells correlated with reduced tumor growth, not enhanced tumorigenesis. To determine the mechanisms underlying this discrepancy, the investigators RNAi-depleted this signaling adaptor in FIP200-null cells to assess how cotargeting these molecules impacted tumor growth. Remarkably, p62 knockdown in FIP200-null tumors further impaired tumor growth compared with FIP200 alone. Thus, in certain circumstances, autophagy synergizes with p62 to promote tumor cell survival or expansion. To validate this result, Wei et al. (2014) performed a rescue of p62 expression in p62-deficient, FIP200-null tumor cells, which led to a partial restoration of tumor growth. Collectively, these observations highlight a previously unknown collaboration between the FIP200 pathway and p62 that can promote the growth of established tumors.

Although the observations of Wei et al. (2014) suggest that autophagy synergizes with p62 to drive tumorigenesis, 
it is conceivable that they merely highlight a unique relationship between FIP200 and tumor growth. Hence, the investigators analyzed the effects of autophagy upregulation on tumor growth by conditionally overexpressing a constitutively active form of the transcription factor $\mathrm{EB}\left(\mathrm{TFEB}^{\mathrm{S} 142 \mathrm{~A}}\right)$, a master transcriptional regulator of molecules that coordinately promote both autophagy and lysosome biogenesis (Settembre et al. 2013). Congruent with a protumorigenic role for autophagy in this system, TFEB ${ }^{\mathrm{S} 142 \mathrm{~A}}$ overexpression significantly enhanced the growth and survival of tumor cells in vivo. However, given the known effects of TFEB ${ }^{\mathrm{S} 142 \mathrm{~A}}$ on lysosome biogenesis, it remains unclear whether enhanced tumor progression is the specific result of enhanced autophagy.

The precise mechanism by which $\mathrm{p} 62$ promotes growth in the absence of autophagy is a point of significant importance, particularly because dual targeting of autophagy and p62 may be an effective treatment for some cancers. To provide mechanistic insight into the tumorpromoting function of p62, Wei et al. (2014) examined the impact of NF-kB suppression on FIP200-null tumor growth. Indeed, p62 has been shown to be necessary for activation of the NF-кB pathway, and these functions have been reported to be important contributors to tumorigenesis (Duran et al. 2008; Mathew et al. 2009). However, expression of the IкB $\alpha$ super repressor, a dominant-negative inhibitor of the NF- $\mathrm{BB}$ pathway, within FIP200-null tumors only modestly reduced tumor growth. Thus, other functions of p62 are also likely to be important for its protumorigenic role in the context of FIP200 ablation. One intriguing possibility is that accumulation of $\mathrm{p} 62$ promotes tumor growth by potentiating mTOR signaling (Fig. 1). Recently, p62 was found to facilitate the activation of mTORC1 by promoting its ubiquitination and lysosomal translocation (Duran et al. 2011; Linares et al. 2013). Given the role of mTOR in coordinating cell growth and its frequent dysregulation in cancer, it is attractive to speculate that the tumor-promoting functions of p62 may be partly rooted in this pathway. In addition, p62 regulation of NRF2-KEAP1 and the antioxidant defense pathway may also facilitate tumorigenesis in the absence of functional FIP200 (Fig. 1; DeNicola et al. 2011). Clearly, the tumorpromoting mechanisms of p62 in autophagy-deficient cells remains a critical area of future investigation.

Finally, it is important to consider the therapeutic potential of simultaneously targeting autophagy and p62 within established cancers. Although Wei et al. (2014) justified such a combinatorial approach in certain scenarios, ultimately, the availability of pharmacological inhibitors to efficiently target these pathways and further understanding of the contexts that maximize treatment efficacy will govern its therapeutic utility. In the first regard, pharmacological autophagy inhibitors such as hydroxychloroquine are already in clinical trials (Yang et al. 2011). In contrast, p62 inhibitors still need to be developed. Nevertheless, the advent of personalized medicine and an explosion in capabilities in GEMMs should make the task of identifying cancer subtypes and stages that are suitably targeted by autophagy and p62 inhibitors far less challenging than it once appeared. Gradually, we are "stacking the deck" with therapeutic options to most effectively target autophagy for cancer treatment.

\section{Acknowledgments}

Grant support to J.D. includes the National Institutes of Health (R01CA126792 and CA126792-S1), the Department of Defense Breast Cancer Research Program (W81XWH-11-1-0130 and W81XWH-12-1-0505), and the Samuel Waxman Cancer Research Foundation.

\section{References}

Boya P, Reggiori F, Codogno P. 2013. Emerging regulation and functions of autophagy. Nat Cell Biol 15: 713-720.

DeNicola GM, Karreth FA, Humpton TJ, Gopinathan A, Wei C, Frese K, Mangal D, Yu KH, Yeo CJ, Calhoun ES, et al. 2011. Oncogene-induced Nrf2 transcription promotes ROS detoxification and tumorigenesis. Nature 475: 106-109.

Duran A, Linares JF, Galvez AS, Wikenheiser K, Flores JM, DiazMeco MT, Moscat J. 2008. The signaling adaptor p62 is an important NF-кB mediator in tumorigenesis. Cancer Cell 13: 343-354.

Duran A, Amanchy R, Linares JF, Joshi J, Abu-Baker S, Porollo A, Hansen M, Moscat J, Diaz-Meco MT. 2011. p62 is a key regulator of nutrient sensing in the mTORC1 pathway. Mol Cell 44: 134-146.

Guo JY, Xia B, White E. 2013. Autophagy-mediated tumor promotion. Cell 155: 1216-1219.

Kimmelman AC. 2011. The dynamic nature of autophagy in cancer. Genes Dev 25: 1999-2010.

Linares JF, Duran A, Yajima T, Pasparakis M, Moscat J, DiazMeco MT. 2013. K63 polyubiquitination and activation of mTOR by the p62-TRAF6 complex in nutrient-activated cells. Mol Cell 51: 283-296.

Mathew R, Karp CM, Beaudoin B, Vuong N, Chen G, Chen H-Y, Bray K, Reddy A, Bhanot G, Gelinas C, et al. 2009. Autophagy suppresses tumorigenesis through elimination of $\mathrm{p} 62$. Cell 137: 1062-1075.

Settembre C, Fraldi A, Medina DL, Sinicrope FA. 2013. Signals from the lysosome: a control centre for cellular clearance and energy metabolism. Nat Rev Mol Cell Biol 14: 283-296.

Wei H, Wei S, Gan B, Peng X, Zou W, Guan J-L. 2011. Suppression of autophagy by FIP200 deletion inhibits mammary tumorigenesis. Genes Dev 25: 1510-1527.

Wei H, Wang C, Croce CM, Guan J-L. 2014. p62/SQSTM1 synergizes with autophagy for tumor growth in vivo. Genes Dev (this issue). doi: 10.1101/gad.237354.113.

White E. 2012. Deconvoluting the context-dependent role for autophagy in cancer. Nat Rev Cancer 12: 401-410.

Yang ZJ, Chee CE, Huang S, Sinicrope FA. 2011. The role of autophagy in cancer: therapeutic implications. Mol Cancer Ther 10: 1533-1541. 


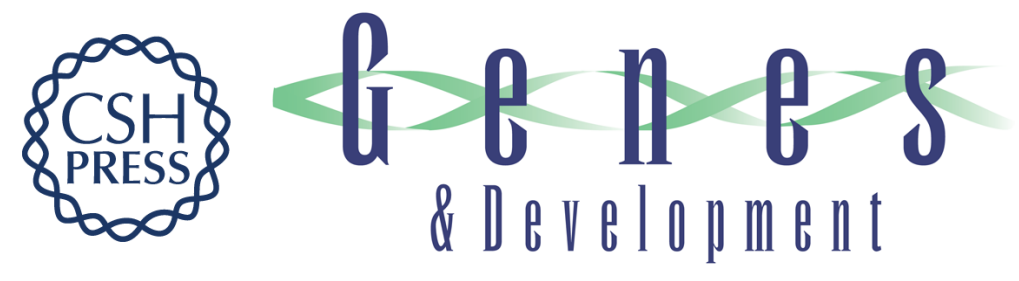

\section{'Doubling down' on the autophagy pathway to suppress tumor growth}

Andrew M. Leidal and Jayanta Debnath

Genes Dev. 2014, 28:

Access the most recent version at doi:10.1101/gad.244681.114

\section{Related Content}

References

Creative
Commons
License

Email Alerting
p62/SQSTM1 synergizes with autophagy for tumor growth in vivo Huijun Wei, Chenran Wang, Carlo M. Croce, et al. Genes Dev. June , 2014 28: 1204-1216

This article cites 12 articles, 3 of which can be accessed free at: http://genesdev.cshlp.org/content/28/11/1137.full.html\#ref-list-1

Articles cited in:

http://genesdev.cshlp.org/content/28/11/1137.full.html\#related-urls

This article is distributed exclusively by Cold Spring Harbor Laboratory Press for the first six months after the full-issue publication date (see

$\mathrm{http}: / / g e n e s d e v . c s h l p . o r g / s i t e / m i s c / t e r m s . x h t m l)$. After six months, it is available under a Creative Commons License (Attribution-NonCommercial 4.0 International), as described at http://creativecommons.org/licenses/by-nc/4.0/.

Receive free email alerts when new articles cite this article - sign up in the box at the top right corner of the article or click here.

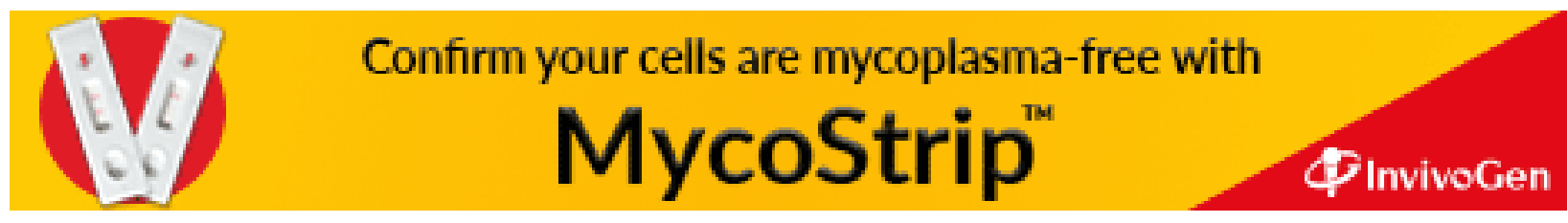

